Original research article

\title{
Control over spectral hole burning via spontaneously generated coherence and Kerr non-linearity
}

\author{
Hazrat Ali $^{\text {a, } * \text {, M. Imtiaz Khan }}{ }^{\mathrm{a}}$, Muhammad Zubair ${ }^{\mathrm{b}}$, Pervaiz Ahmad ${ }^{\mathrm{c}}$, \\ Mayeen Uddin Khandaker ${ }^{\mathrm{d}}$, Tariq Ali ${ }^{\mathrm{e}}$, Farooq Khan ${ }^{\mathrm{f}}$ \\ ${ }^{a}$ Department of Physics, Abbottabad University of Science and Technology, Havelian, Pakistan \\ ${ }^{\mathrm{b}}$ College of Materials Science and Engineering, Key Laboratory of Advance Functional Material, Ministry of Education, Beijing University of \\ Technology, Beijing 1000124, China \\ ${ }^{\mathrm{c}}$ Department of Physics, University of Azad Jammu \& Kashmir, Muzaffarabad, 13100, Pakistan \\ ${ }^{\mathrm{d}}$ Center for Biomedical Physics, School of Healthcare and Medical Sciences, Sunway University, Bandar Sunway, Selangor, 47500, Malaysia \\ e Department of Physics, United Arab Emirates University, Al Ain, United Arab Emirates \\ ${ }^{\mathrm{f}}$ Department of Physics Balochistan University of Information Technology, Engineering and Management Sciences, Quetta, Pakistan
}

\section{A R T I C L E I N F O}

\section{Keywords:}

Electromagnetically induced transparency

Kerr Field

Spectral hole burning

\begin{abstract}
A B S T R A C T
We study the control over spectral hole burning in four-level N-type system via spontaneously generated coherence (SGC) and Kerr nonlinearity in an inhomogeneous Doppler broadening medium. We consider a co-propagating probe laser beam and a counter-propagating control laser beam at the same time. Multiple spectral holes are created with the spontaneously generated coherence in the medium. The depth of the spectral holes gets deeper with increasing of the Kerr field. We study the group index and the transmission of probe laser pulse in SHB regions that lead us to achieve maximum transmission and much steeper group index in the system with increasing the strength of the Kerr field.
\end{abstract}

\section{Introduction}

Quantum coherence is an effective technique used to manipulate the optical properties of the atomic medium. Electromagnetically induced transparency (EIT) [1,2] is an optical phenomenon arises from the interference between the transition of two dress states [3]. Coherent based amplification of light has been investigated in Double EIT medium [4]. Cai et al. have measured experimentally the chiral edge current of atoms by employing two coherent standing waves in $\Lambda$ type atomic medium at room temperature [5]. Spin Hall effect of light can be controlled and enhanced in multilevel atomic medium under coherent effect [6]. An ultra-slow light [7-12], lasing without inversion [12,13], optical memory [14-16] and optical hole burning (HB) [17-21] are the outcomes of the atomic coherence. Kuang et al. have studied the Coherent HB to achieve a low group velocity of the light pulse in three-level Doppler broadening atomic medium [22] and Tian et al. have noticed six spectral HBs in the absorption spectrum of the four level Doppler broadened medium [23]. Also, slow light with persistent HB has been studied in Refs. [18,19]. Recently, Putz et al. have demonstrated experimentally the bleaching of the ensemble's absorption spectrum in hybrid quantum systems and thus the generated holes create the long-lived dark states [20]. Furthermore, Debnath et al. employed the technique of stimulated Raman adiabatic passage (SRAP) in three-level lambda system and found the narrow absorption regions inside the burned hole. These narrow regions within a broad ensemble can be serve as

\footnotetext{
* Corresponding author.

E-mail addresses: yamanuom@gmail.com (H. Ali), muimtiazkhan@gmail.com (M.I. Khan).
} 